\title{
Formation and Evolution Mechanism of Plasmon Resonance from Single Ring-Shaped Nanotube to Dimer and Arrays
}

\author{
F. Q. Zhou, ${ }^{1}$ Z. M. Liu, ${ }^{1,2}$ H. J. Li, ${ }^{2}$ Z. F. Liu, ${ }^{1}$ B. Tang, ${ }^{3}$ and X. Zhou ${ }^{4}$ \\ ${ }^{1}$ College of Sciences, East China Jiaotong University, Nanchang 330013, China \\ ${ }^{2}$ College of Physics Science and Technology, Central South University, Changsha 410083, China \\ ${ }^{3}$ School of Mathematics and Physics, Changzhou University, Changzhou 213164, China \\ ${ }^{4}$ School of Sciences, Hunan University of Technology, Zhuzhou 412008, China
}

Correspondence should be addressed to Z. M. Liu; liuzhimin2006@163.com

Received 8 October 2013; Accepted 7 January 2014; Published 2 April 2014

Academic Editor: Clare C. Byeon

Copyright (C) 2014 F. Q. Zhou et al. This is an open access article distributed under the Creative Commons Attribution License, which permits unrestricted use, distribution, and reproduction in any medium, provided the original work is properly cited.

\begin{abstract}
The formation and evolution mechanisms of plasmon resonance from single Ring-shaped nanotube to dimer and arrays are studied; an attempt has been made to bridge the gap between single-tube, dimer, and array. Results show that resonant modes can be divided into three types: quadrupole, hexapole, and octupole resonance from visible to near infrared region, and each mode maintains relatively stable resonant characteristics, but the optical transmission properties including redshift and blueshift of the modes and band gap are highly tunable by adjusting the number of nanotube and intertube spacing values. The field-interference mechanism has been suggested to explain the physical origin.
\end{abstract}

\section{Introduction}

Surface plasmon (SP) stems from the coupling between metallic nanostructures and light and great interest has been aroused for its rich physical phenomena, mechanisms, and potential application [1-4].

The studies on single metallic nanostructures include nanospheres [5], nanoprisms [6], nanorods [7], nanocubes [8], and shells [9]. The results show that the plasmonic properties depend strongly on the size and shape of the nanostructure and material properties of the surrounding medium.

Coupled metallic nanoparticle pairs are referred to as plasmonic "dimer" [10]. The effects of symmetry breaking on plasmon resonance of dimer have been analyzed in many works [11, 12], and the "hot spots" may be formed by the pair of closely spaced nanoparticles and caused by the strong coupling interactions between the near fields of two closely spaced nanoparticles $[11,13]$.

A nanosystem such as nanowire with nanoparticle [14, 15], nanoparticles array [16], and nanowires chain systems $[17,18]$ is interesting for the interactions of plasmon modes between the individual nanostructures [19]. In addition, arrays of nanostructures can also be used as optical waveguides [20-22]. Many efforts focused on the optical transmission properties of simple nanoparticles such as spherical particles and nanotube arrays [23-28].

Single nanostructure, dimer, and arrays have been highly studied, respectively. And the above researched nanostructures such as circular-shell-core nanoparticle or nanotube are the common structure; the metallic Ring-shaped nanotube and its array have not been discussed comprehensively; and the existence of length $L$ of the Ring-shaped nanotube (shape of nanotube) influences greatly the transmission spectrum and plasmon resonance. In addition, we want to research the formation and evolution mechanisms of plasmon resonance between single particle, dimer, and array.

Based on the above analysis, in this paper, we propose single Ring-shaped nanotube, dimer, and arrays and discuss its optical transmission properties by using Finite-Difference Time-Domain (FDTD) method. First, we make a simple comparison between the Circular-shaped and Ring-shaped nanotube arrays. Then, from the transmission spectra of the simulation results, we can see the formation and evolution 
process of the regularly resonant transmission modes and band gap. A field-interference mechanism has been suggested and investigated. In addition, the transmission properties have been further characterized with various intertube spacing between adjacent nanotubes. The present study has led to an improved understanding of the formation and evolution of plasmon resonant modes in nanostructures, and the proposed models and results may have great potential applications in subwavelength near-field optics.

\section{Model and Method}

Figure 1 shows schematically two-dimensional Ringshaped nanotube with infinite length along the $z$ direction. Figure 1(a) is the single-tube, in the whole paper, the outer radius, inner radius, and the length of the Ring-shaped nanotube are fixed $R_{1}=50 \mathrm{~nm}, R_{2}=40 \mathrm{~nm}$, and $L=100 \mathrm{~nm}$, respectively. A nanodimer composed by two nanotube pairs is shown in Figure 1(b), and the intertube spacing between adjacent nanotubes can be changed by varying the parameter $D$. Further, the array of ten nanotubes is depicted in Figure 1(c). In addition, the metal is chosen to be Au. The relative permittivities of the gold are approximated by the Drude model, which is defined as $\varepsilon(\omega)=1-\omega_{p}^{2} /\left(\omega^{2}+i \omega \gamma_{p}\right)$, where $\omega_{p}=1.37 \times 10^{16} \mathrm{~s}^{-1}$ is the bulk plasmon frequency and $\gamma_{p}=4.08 \times 10^{13} \mathrm{~s}^{-1}$ represents the electron relaxation time [29]. The outside and inner-core outside dielectric medium of Ring-shaped nanotube are defined as air; the dielectric constant is $\varepsilon=1$.

We use two-dimensional FDTD [30] method to simulate the structure with a lattice of $L_{x} \times L_{y}=1800 \mathrm{~nm} \times 300 \mathrm{~nm}$ for single-tube, dimer, and array, and infinity along the $z$ direction, and the spatial mesh and time steps are set as $\Delta_{x}=$ $\Delta_{y}=1 \mathrm{~nm}$ and $\Delta t=\Delta x / 2 c$, where $c$ is the velocity of light in vacuum. The left and right surfaces of the calculated region along the $x$ direction are truncated by perfectly matched layer (PML) absorbing boundary conditions, and due to the periodicity of the structure along $y$ direction, the top and bottom boundaries along $y$ direction are treated by periodic boundary conditions. A Gaussian single pulse of light with a wide frequency profile is sent along the $x$ direction with TM polarization.

\section{The Differences between the Circular- Shaped and Ring-Shaped Nanotube Arrays}

Let us make a simple comparison between the Circularshaped and Ring-shaped nanotube arrays. We fix the outer radius $R_{1}=50 \mathrm{~nm}$, inner radius $R_{2}=40 \mathrm{~nm}$, and separation distance of tubes $D=20 \mathrm{~nm}$ both for Circular-shaped and Ring-shaped nanotube arrays and set length $L=100 \mathrm{~nm}$ of the Ring-shaped nanotube.

The transmission spectrum of the band structure can be formed in both the Circular-shaped and the Ring-shaped nanotube arrays. From Figure 2(a), we can see that only one forbidden band has been formed in range from $428 \mathrm{~nm}$ to $577 \mathrm{~nm}$, and it shows that the resonant peaks on the left side are dense and irregular, while the resonant peaks on the right side are regular. While two forbidden bands form intransmission spectrum of the Ring-shaped nanotube array, which is showed in Figure 2(b), the right forbidden band is very wide ranging from $680 \mathrm{~nm}$ to $1000 \mathrm{~nm}$, the left forbidden band is narrow ranging from $370 \mathrm{~nm}$ to $500 \mathrm{~nm}$, and five regular resonant peaks appear between the two forbidden bands. From the results, we can find that the existence of length $L$ of the Ring-shaped nanotube influence greatly the transmission spectrum and plasmon resonance; the details of resonant properties of Ring-shaped nanotube are studied in next sections. This is why we choose this structure.

\section{Numerical Results and Discussion: Formation and Evolution Mechanisms of Plasmon Resonance}

First of all, we fix the outer radius $R_{1}=50 \mathrm{~nm}$, inner radius $R_{2}=40 \mathrm{~nm}$, and separation distance of tubes $D=20 \mathrm{~nm}$ and discuss the effect of the tube number $N$ on optical transmission properties and then study the formation and evolution mechanisms of plasmon resonance from single Ring-shaped nanotube to dimer and array. The transmission spectrum with different numbers $N=1,2,10$ are shown in Figures 3(a)-3(c), namely, the single-tube, dimer, and array, respectively. We find some features by comparing Figures 3(a)-3(c). First, the resonant modes can be grouped into three types. Type 1 is quadrupole resonance including the peaks of $\mathrm{A} 1, \mathrm{~A} 2$, and $\mathrm{A} 3$, and the Type 2 peaks B1, B2, and B3 belong to hexapole resonance; the Type 3, C1, C2, and C3, is octupole resonance; we will discuss the multipole resonant properties by simulating the electric field distributions in next chapter. Second, from Figure 3, we can find that the properties of the three types of modes exhibit certain regularity with $N$ increasing. When only the single-tube system is considered, the higher-order modes are excited in the visible and near infrared region, just like the resonant peaks B1 and C1, while the quadrupole resonance peak A1 stays away from this area and distributes in infrared region (not shown here). Then, we take into account the dimer system; due to the coupling effect between nanotube pairs, quadrupole resonance peak A2 gradually forms in near infrared region; we also consider that quadrupole resonance peak A2 blueshifts into near infrared region. At the same time, the higher-order modes $\mathrm{B} 1$ and $\mathrm{C} 1$, the dip between $\mathrm{B} 1$ and $\mathrm{C} 1$, and the dip on the right $\mathrm{B} 1$ redshift. It is more important that the transmission band begins to form; forbidden band can clearly be seen in the transmission spectra from Figure 3(b). As shown in Figure 3(c), due to the coupling effect between each nanotube, regular quadrupole resonance peak A3 and hexapole resonance peak B3 establish; for example, six hexapole resonance peaks B3 appear. In addition, two wide forbidden bands have been formed, the right forbidden band ranges from $680 \mathrm{~nm}$ to $1000 \mathrm{~nm}$, the left band gap is smaller than the right, and it ranges from $380 \mathrm{~nm}$ to $500 \mathrm{~nm}$. The formation and evolution process is similar to the forming process of band lines in photonic crystal, which can be used to design an optical filter.

In order to analyse the basic physical mechanisms for above phenomena, we plot the electric field distributions $E_{x}$ 


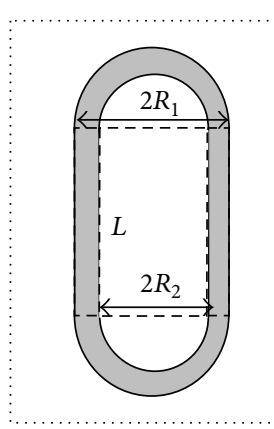

(a) Single-tube

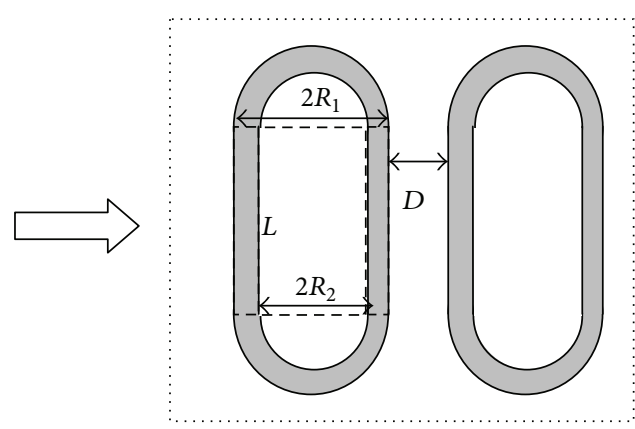

(b) Dimer

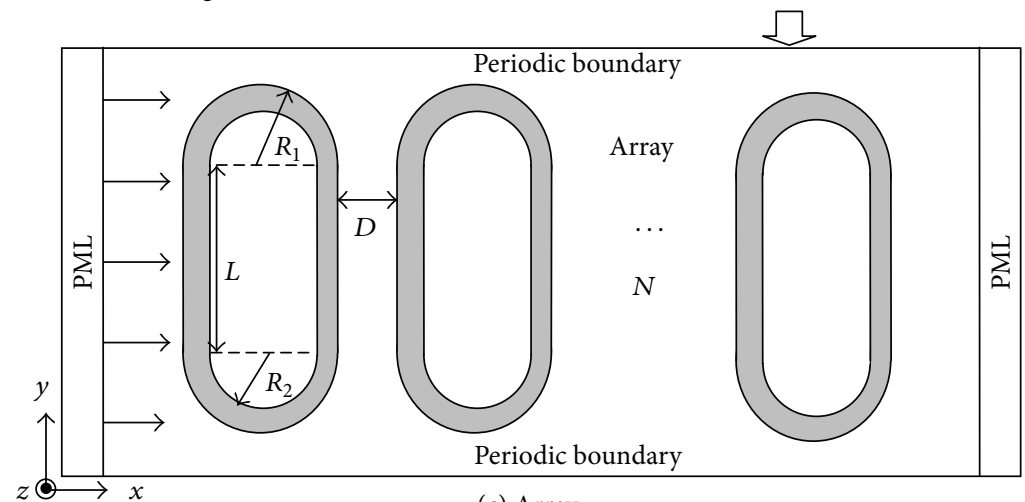

(c) Array

FIgURe 1: A unit cell of the Ring-nanotube (a) single-tube; (b) dimer; (c) array.

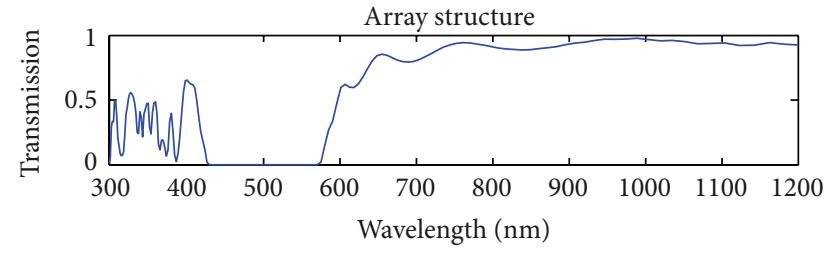

(a)

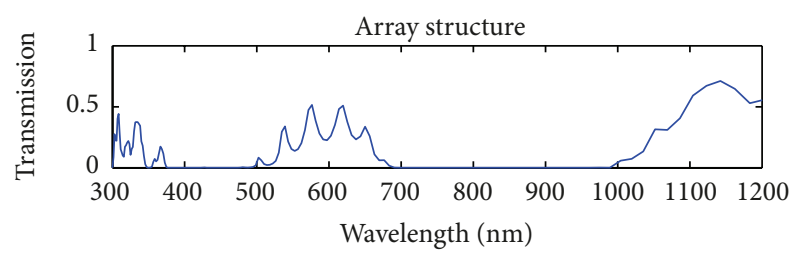

(b)

FIgURE 2: Transmission spectra through the Circular-shaped (a) and Ring-shaped (b) nanotube arrays as a function of wavelength.

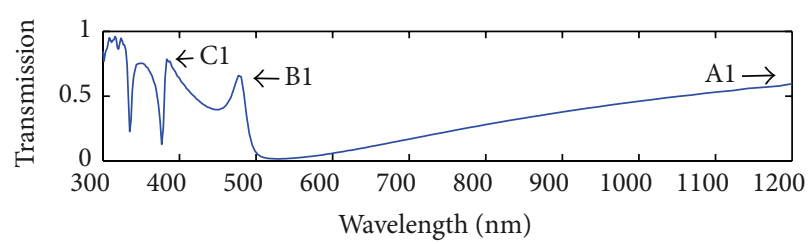

(a)

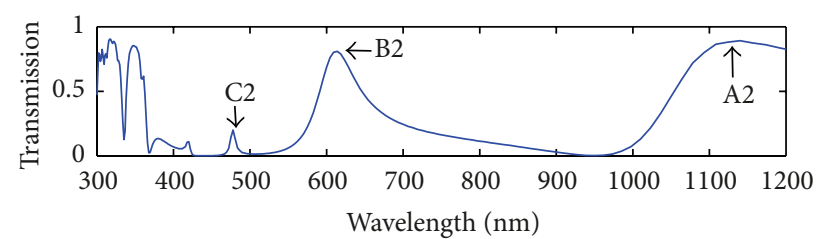

(b)

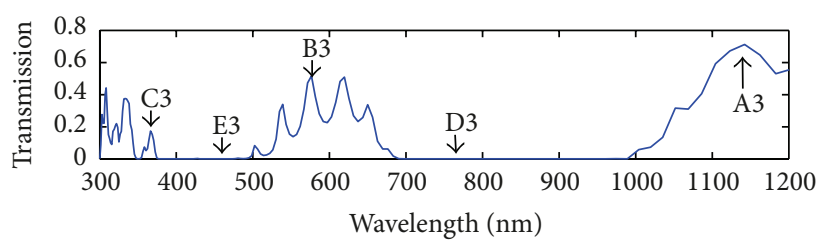

(c)

FIgURE 3: Transmission spectra for single-tube (a); dimer (b); array (c). 


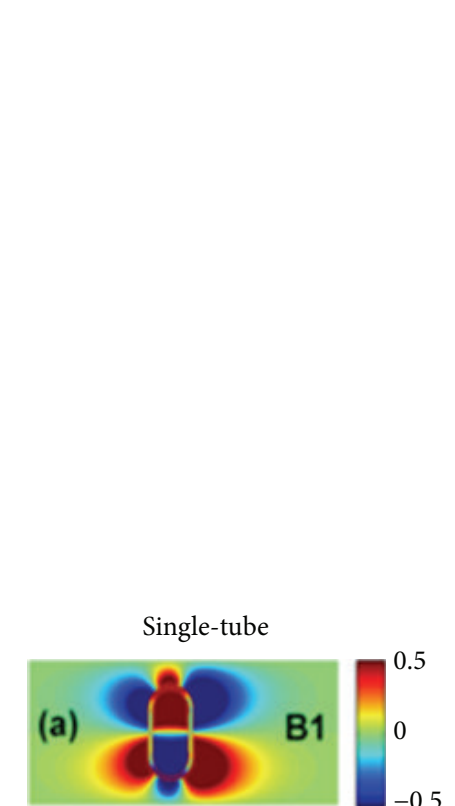

(a)

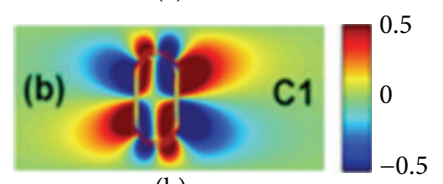

(b)

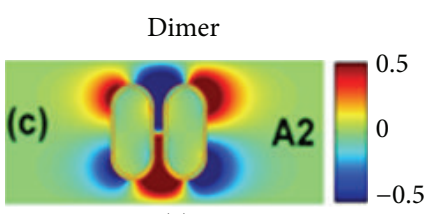

(c)

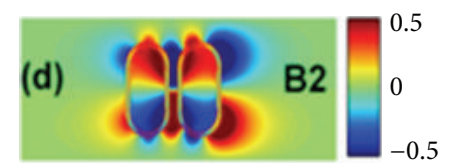

(d)

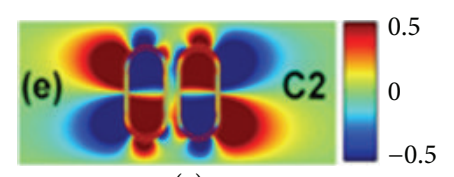

(e)

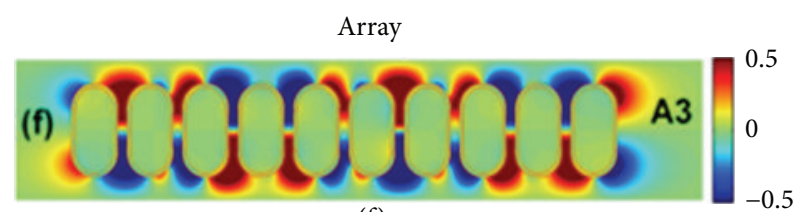

(f)

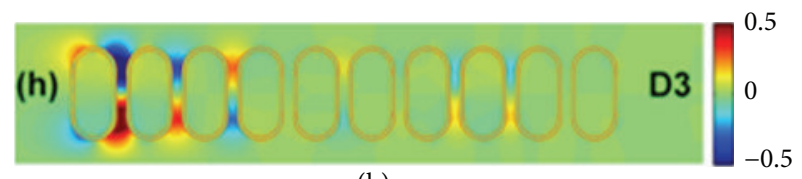

(h)

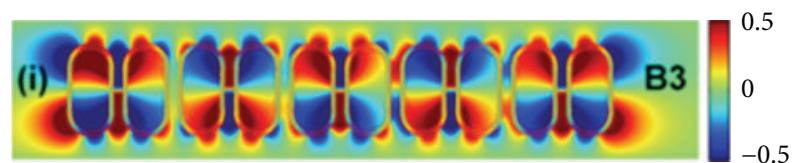

(i)

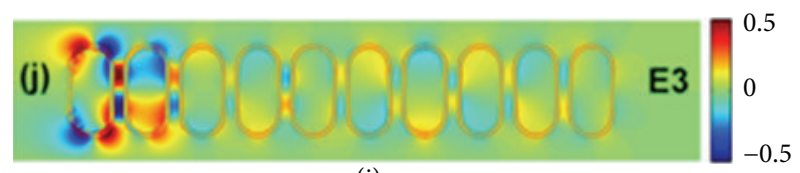

(j)

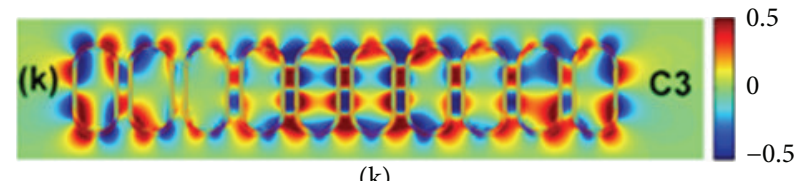

(k)

Figure 4: The field distributions of $E_{x}$ at the transmission peaks and band gap (A, B, C, D, and E) are labeled in Figure 2, left column for single-tube, middle column for dimer, and right column for array.

at the transmission peaks $\mathrm{B} 1$ and $\mathrm{C} 1$ for single-tube in Figures 4(a) and 4(b); transmission peaks A2, B2, and C2 for dimer in Figures 4(c)-4(e) and transmission peaks A3, B3, and C3 and band gap D3 and E3 for array in Figures 4(f)-4(k) and the corresponding peaks have been labeled in Figure 3, respectively. We consider that red colored areas represent positive and blue means negative.

First, we can find that positive and negative electric field $E_{x}$ spread alternately near the edges of the nanotube from the left column of Figures 4(a) and 4(b). Figure 4(a) shows that the left sign of $E_{x}$ is the same of the right, while they are opposite on the top and bottom for single nanotube, and electric field also exists in the middle and inner of the tube; its sign is opposite to the left and right. We consider that it is a hexapole field distribution for the plasmon modes in the nanotubes. It is the reason that we regard this transmission peaks as the peaks of Type 2 hexapole resonance in Figure 2. In addition, Figure 4(b) depicts the octupole resonance at the Type $3 \mathrm{C} 1$ in Figure 3, but the signs of the top and bottom, left and right, and outside and inner change alternately. Besides, we can see analogous hexapole and octupole resonant patterns in the dimer and array, which are shown in Figures 4(d) and 4(e) for B2 and C2 and Figures 4(i) and 4(k) for B3 and C3. The only difference is that some of the nanotubes form a group; this phenomenon is particularly evident in Figure 4(i) B3, where each of the two tubes forms a group, and five groups appear totally.
Second, Figure 4(c) for A2 and Figure 4(f) for A3 show the quadrupole resonance; the electric field $E_{x}$ distributes nearly outside of the tube, and we could barely see the electric field inner of the tube. The signs of the surface charges at two sides of the gap are opposite; the electric fields between two nanotubes are coupled together closely and intensified, which is just in analogy with a simple capacity device, so the field distributions are mostly concentrated at every other gap between adjacent nanotubes [24-26]. The results indicate that the oscillation of charge density exists between the nanotubes. In other words, the surface plasmon wave really exists and propagates along the surface. Due to the nanotube with different lengths that may be used as chain waveguides, the understanding of the plasmonic behaviors in coupled nanotubes of arrays is especially important [24].

Last, we draw the spatial distributions of the $E_{x}$ at wavelengths of band gap D3 and E3, which are depicted in Figures 4(h) and 4(j); electric field only emerges on left several tubes. No excitation of resonant mode exists owing to the forbidden band gap, and incident light cannot transmit.

Successively, we investigate the effect of different spacing intertube on transmission spectrum and electric field distributions.

Firstly, the transmission spectra varying intertube spacing between adjacent nanotubes for dimer is shown in Figure 5(a). The transmission spectra blueshift obviously with increasing of the intertube spacing; the peak intensities of $\mathrm{A}$ 


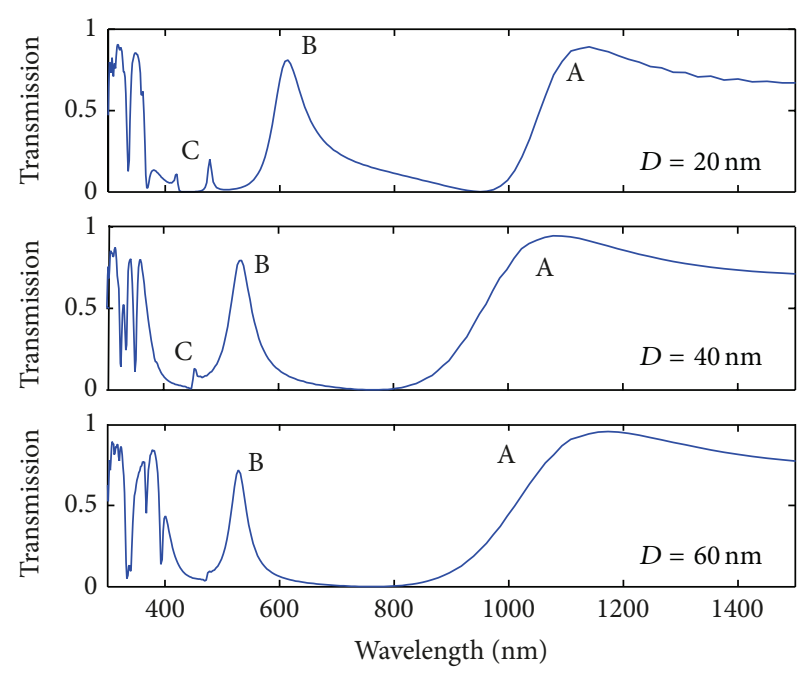

(a)

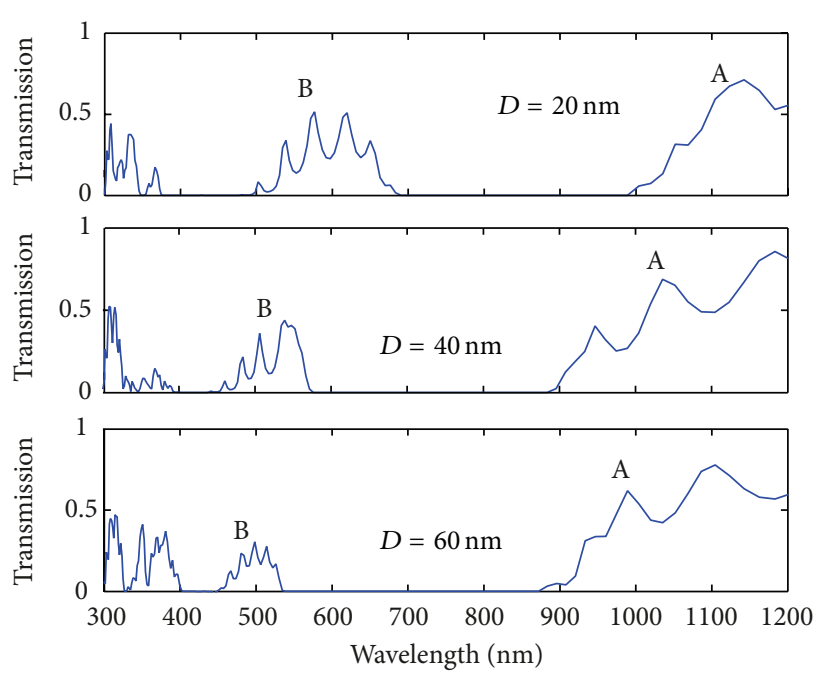

(b)

FIgURE 5: Transmission spectra with different intertube spacing in (a) dimer and (b) array.

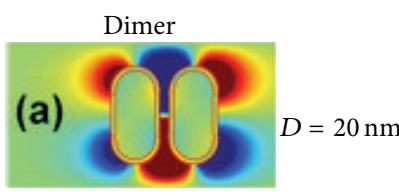

(a)

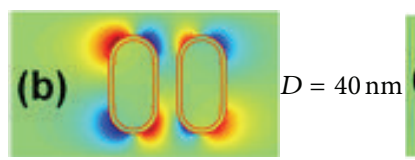

(b)

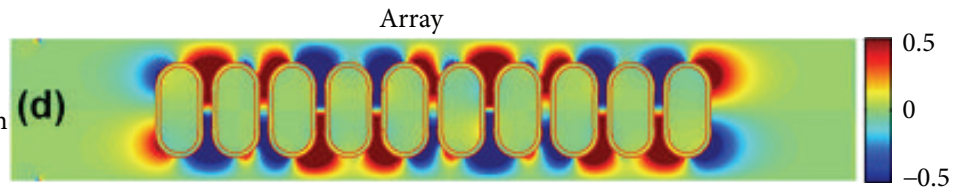

(d)

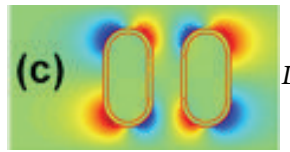

(c)

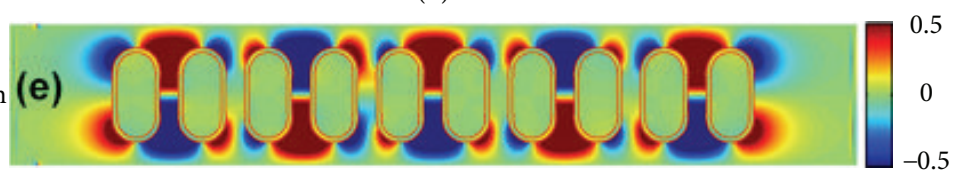

(e) 
four groups form as $D=60 \mathrm{~nm}$. Due to the group formation, the electric fields $E_{x}$ couple strongly with inner of each group, so we do not see that the intensity of electrical field inner of the group attenuates, but electrical field between the group attenuates evidently.

\section{Conclusion}

In conclusion, we proposed single Ring-shaped nanotube, dimer, and arrays and discussed its optical transmission properties; an attempt has been made to bridge the gap between single particle dimer, and array. Results show that the resonant modes can be divided into three types: quadrupole, hexapole, and octupole resonance from the visible to near infrared region, and each mode maintains relatively stable resonant characteristics, but the resonant transmission sensitively depends on the number of nanotubes; magnitude modification, redshift and blueshift of the resonance modes, and band gap are observed. From the transmission spectra and electric field distributions of the single nanotube to dimer and arrays, we can see the formation and evolution process of the regularly resonant transmission modes and band gap. In addition, the transmission properties of the dimer and array have been further characterized with various intertube spacing between adjacent nanotubes. The presented study not only sharpens our understanding of the formation and evolution of plasmon resonant modes in nanostructures but also provides a profound comprehension into the plasmonic interference. And we hope that the proposed models and results can be used to design structures with optimized nanophotonic devices.

\section{Conflict of Interests}

The authors declare that there is no conflict of interests regarding the publication of this paper.

\section{Acknowledgments}

This work was funded by the National Natural Science Foundation of China (Grants nos. 11164007, 61275174, and 61107055), the Natural Science Foundation of Jiangxi province (Grant no. 20132BAB212007), and Hunan Province of China (Grant no. 14JJ3122).

\section{References}

[1] P. K. Jain and M. A. El-Sayed, "Noble metal nanoparticle pairs: effect of medium for enhanced nanosensing," Nano Letters, vol. 8, pp. 4347-4352, 2008.

[2] N. Felidj, J. Aubard, and G. Levia, "Optimized surface-enhanced Raman scattering on gold nanoparticle arrays," Applied Physics Letters, vol. 82, p. 3095, 2003.

[3] C. Hagglund, M. Zach, G. Petersson, and B. Kasemo, "Electromagnetic coupling of light into a silicon solar cell by nanodisk plasmons," Applied Physics Letters, vol. 92, Article ID 053110, 2008.

[4] C. B. Murray, S. Sun, H. Doyle, and T. Betley, "Monodisperse 3d transition-metal ( $\mathrm{Co}, \mathrm{Ni}, \mathrm{Fe})$ nanoparticles and their assembly into nanoparticle superlattices," MRS Bulletin, vol. 26, no. 12, pp. 985-991, 2001.

[5] K. L. Kelly, E. Coronado, L. L. Zhao, and G. C. Schatz, "The optical properties of metal nanoparticles: the influence of size, shape, and dielectric environment," Journal of Physical Chemistry B, vol. 107, no. 3, pp. 668-677, 2003.

[6] K. Munechika, J. M. Smith, Y. C. Chen, and D. S. Ginger, "Plasmon line widths of single silver nanoprisms as a function of particle size and plasmon peak position," Journal of Physical Chemistry C, vol. 111, no. 51, pp. 18906-18911, 2007.

[7] W. H. Ni, X. S. Kou, Z. Yang, and J. F. Wang, "Tailoring longitudinal surface plasmon wavelengths, scattering and absorption cross sections of gold nanorods," ACS Nano, vol. 2, no. 4, pp. 677-686, 2008.

[8] L. J. Sherry, S.-H. Chang, G. C. Schatz, R. P. Van Duyne, B. J. Wiley, and Y. Xia, "Localized surface plasmon resonance spectroscopy of single silver nanocubes," Nano Letters, vol. 5, no. 10, pp. 2034-2038, 2005.

[9] C. M. Aguirre, T. R. Kaspar, C. Radloff, and N. J. Halas, "CTAB mediated reshaping of metallodielectric nanoparticles," Nano Letters, vol. 3, no. 12, pp. 1707-1711, 2003.

[10] N. J. Halas, S. Lal, W.-S. Chang, S. Link, and P. Nordlander, "Plasmons in strongly coupled metallic nanostructures," Chemical Reviews, vol. 111, no. 6, pp. 3913-3961, 2011.

[11] B. Yun, Z. Wang, G. Hu, and Y. Cui, “Theoretical studies on the near field properties of non-concentric core-shell nanoparticle dimers," Optics Communications, vol. 283, no. 14, pp. 2947-2952, 2010.

[12] H. Q. Xu, H. J. Li, Z. M. Liu et al., "Effects of symmetry breaking on plasmon resonance in a noncoaxial nanotube and nanotube dimer," Journal of the Optical Society of America A: Optics and Image Science, and Vision, vol. 28, no. 8, pp. 1662-1667, 2011.

[13] C. E. Talley, J. B. Jackson, C. Oubre et al., "Surface-enhanced Raman scattering from individual $\mathrm{Au}$ nanoparticles and nanoparticle dimer substrates," Nano Letters, vol. 5, no. 8, pp. 1569-1574, 2005.

[14] H. E. Ruda and A. Shik, "Polarization and plasmon effects in nanowire arrays," Applied Physics Letters, vol. 90, no. 22, Article ID 223106, 2007.

[15] H. S. Chu, W. B. Ewe, W. S. Koh, and E. P. Li, "Remarkable influence of the number of nanowires on plasmonic behaviors of the coupled metallic nanowire chain," Applied Physics Letters, vol. 92, no. 10, Article ID 103103, 2008.

[16] F. Hao and P. Nordlander, "Enhanced tunability and linewidth sharpening of plasmon resonances in hybridized metallic ring/disk nanocavities," Physical Review B, vol. 76, Article ID 245417, 2007.

[17] J. P. Kottmann and O. J. F. Martin, "Retardation-induced plasmon resonances in coupled nanoparticles," Optics Letters, vol. 26, no. 14, pp. 1096-1098, 2001.

[18] Y.-F. Chau, H.-H. Yeh, and D. P. Tsai, "Near-field optical properties and surface plasmon effects generated by a dielectric hole in a silver-shell nanocylinder pair," Applied Optics, vol. 47, no. 30, pp. 5557-5561, 2008.

[19] D. J. Wu, X. D. Xu, and X. J. Liu, "Optimization of silicasilver-gold layered nanoshell for large near-field enhancement," Applied Physics Letters, vol. 96, Article ID 151912, 2010.

[20] Y.-F. Chau, H.-H. Yeh, C.-Y. Liu, and D. P. Tsai, “The optical properties in a chain waveguide of an array of silver nanoshell with dielectric holes," Optics Communications, vol. 283, no. 16, pp. 3189-3193, 2010. 
[21] R. Zia, J. A. Schuller, and M. L. Brongersma, "Near-field characterization of guided polariton propagation and cutoff in surface plasmon waveguides," Physical Review B-Condensed Matter and Materials Physics, vol. 74, no. 16, Article ID 165415, 2006.

[22] A. F. Koenderink, R. De Waele, J. C. Prangsma, and A. Polman, "Experimental evidence for large dynamic effects on the plasmon dispersion of subwavelength metal nanoparticle waveguides," Physical Review B-Condensed Matter and Materials Physics, vol. 76, no. 20, Article ID 201403, 2007.

[23] H. J. Li, Q. Liu, S. X. Xie, X. Zhou, H. Xia, and R. Zhou, "Particle plasmons resonant characteristics in arrays of strongly coupled gold nanoparticles," Solid State Communications, vol. 149, no. 56, pp. 239-242, 2009.

[24] H. J. Li, S. L. Fu, S. X. Xie, S. Xie, X. Zhou, and J. Wu, “The optical transmission characteristics through coupled metallic nanotube arrays," Optics Communications, vol. 283, no. 20, pp. 3985-3988, 2010.

[25] S. L. Fu, H. J. Li, and S. X. Xie, "Tuning of plasmonic behaviours in coupled metallic nanotube arrays," Chinese Physics B, vol. 20, no. 8, Article ID 087302, 2011.

[26] Z. M. Liu, H. J. Li, H. Q. Xu, S. Xie, X. Zhou, and C. Wu, “Tunable optical transmission through square-core metallic nanotube arrays," Optics Communications, vol. 284, no. 13, pp. 3331-3334, 2011.

[27] H. Q. Xu, H. J. Li, and G. Xiao, "Tunable plasmon resonance coupling in coaxial gold nanotube arrays," Chinese Optical Letters, vol. 11, Article ID 042401, 2013.

[28] X. Zhou, J. S. Fang, D. W. Yang, and X. P. Liao, "Plasmon resonance coupling in strongly coupled gold nanotube arrays with structural defects," Chinese Physical B, vol. 21, no. 8, Article ID 084202, 2012.

[29] E. D. Palik, Handbook of Optical Constants in Solids, Academic Press, Boston, Mass, USA, 1982.

[30] A. Taflove and S. C. Hagness, Computational Electrodynamics: The Finite-Difference-Time-Domain Method, Artech House, Boston, Mass, USA, 2nd edition, 2000. 

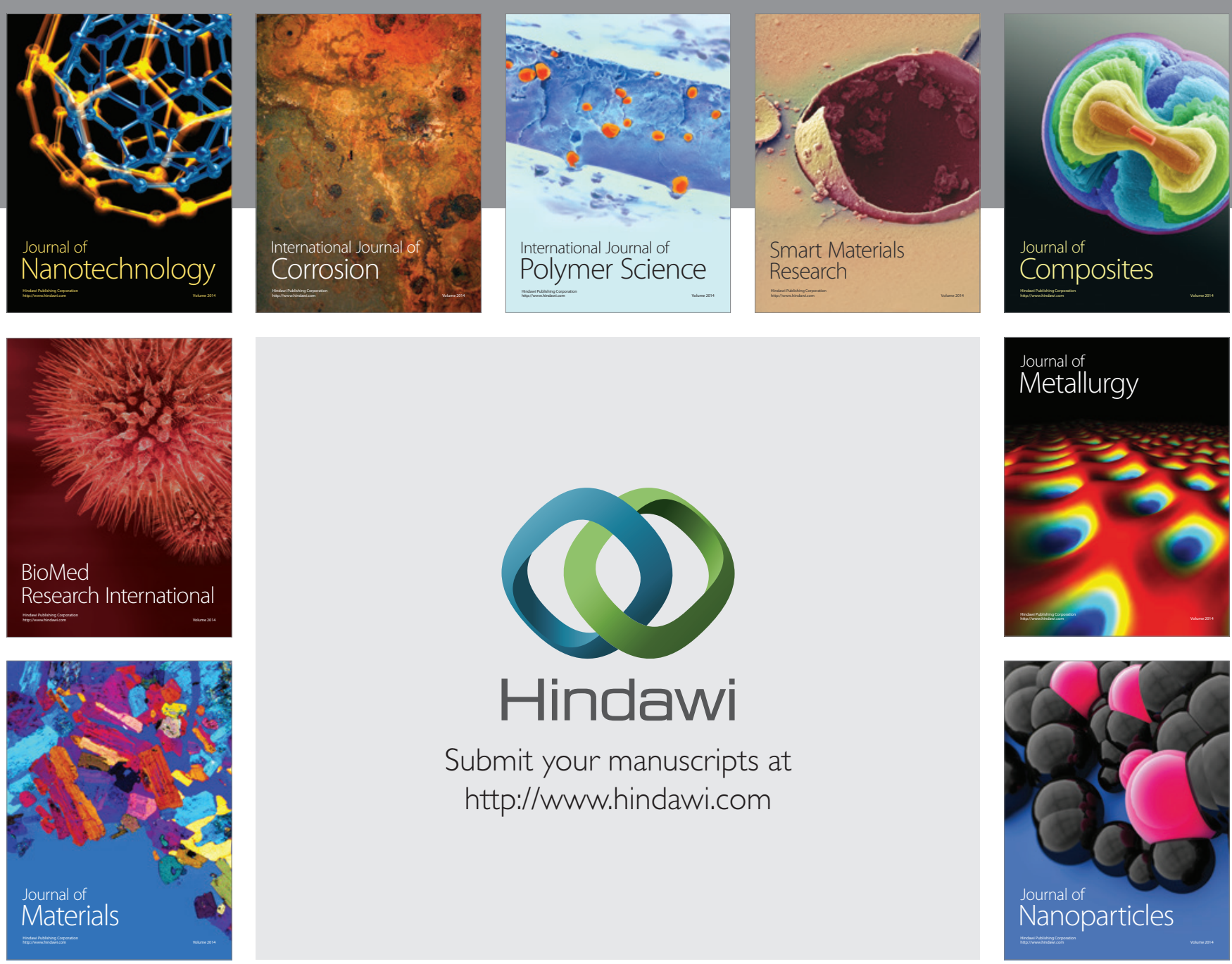

Submit your manuscripts at http://www.hindawi.com
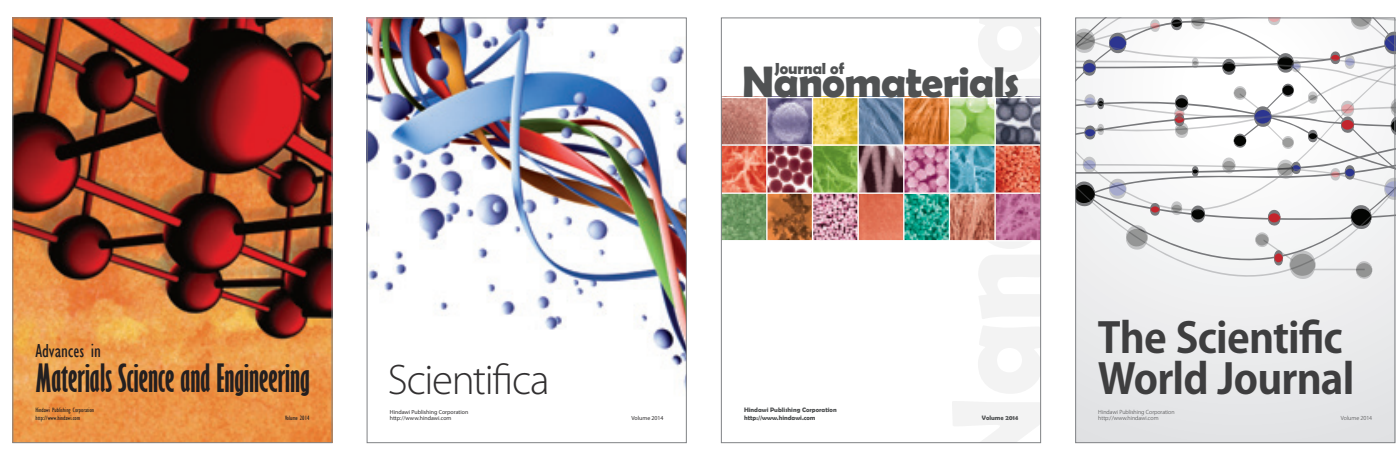

\section{The Scientific World Journal}
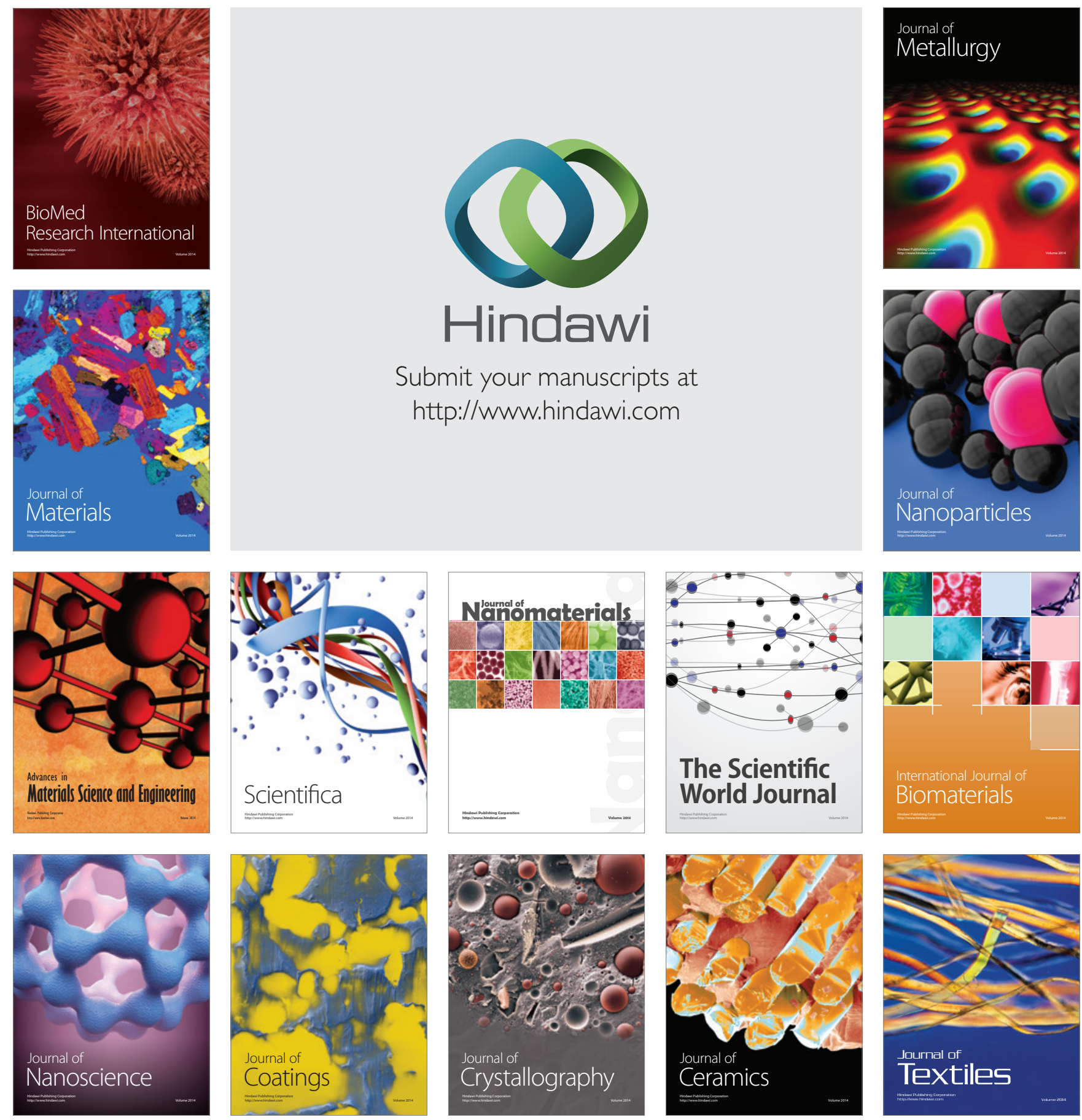